\title{
Use of the Resection Map system as guidance during hepatectomy
}

\author{
Pablo Lamata $\cdot$ Félix Lamata $\cdot$ Valentin Sojar · Piotr Makowski · \\ Laurent Massoptier · Sergio Casciaro • Wajid Ali $\cdot$ Thomas Stüdeli • \\ Jérôme Declerck · Ole Jackov Elle $\cdot$ Björn Edwin
}

Received: 29 April 2009/Accepted: 14 August 2009/Published online: 23 February 2010

(C) The Author(s) 2010. This article is published with open access at Springerlink.com

\begin{abstract}
Background The objective of this work is to evaluate a new concept of intraoperative three-dimensional (3D) visualization system to support hepatectomy. The Resection Map aims to provide accurate cartography for surgeons, who can therefore anticipate risks, increase their confidence and achieve safer liver resection.

Methods In an experimental prospective cohort study, ten consecutive patients admitted for hepatectomy to three European hospitals were selected. Liver structures (portal veins, hepatic veins, tumours and parenchyma) were segmented from a recent computed tomography (CT) study of
\end{abstract}

P. Lamata · J. Declerck

Siemens Molecular Imaging, Oxford, UK

F. Lamata

Hospital Clínico Universitario, Zaragoza, Spain

V. Sojar

University Clinik Center, Ljubljana, Slovenia

P. Makowski - L. Massoptier · S. Casciaro

Institute of Clinical Physiology, Biomedical Engineering

Division, National Council of Research (CNR-IFC), 73100

Lecce, Italy

W. Ali · O. J. Elle · B. Edwin

The Interventional Centre, Oslo University Hospital, Oslo, Norway

T. Stüdeli

Faculty of Industrial Design Engineering, Delft University of Technology, Delft, The Netherlands

P. Lamata $(\bowtie)$

Computing Laboratory, University of Oxford, Wolfson Building,

Parks Road, Oxford OX1 3QD, UK

e-mail: Pablo.lamata@hotmail.com each patient. The surgeon planned the resection preoperatively and read the Resection Map as reference guidance during the procedure. Objective (amount of bleeding, tumour resection margin and operating time) and subjective parameters were retrieved after each case.

Results Three different surgeons operated on seven patients with the navigation aid of the Resection Map. Veins displayed in the Resection Map were identified during the surgical procedure in $70.1 \%$ of cases, depending mainly on size. Surgeons were able to track resection progress and experienced improved orientation and increased confidence during the procedure.

Conclusions The Resection Map is a pragmatic solution to enhance the orientation and confidence of the surgeon. Further studies are needed to demonstrate improvement in patient safety.

Keywords Computer-assisted surgery · Hepatectomy · Instrumentation

Colorectal cancer is the third most common cancer in the USA, with an estimated 150,000 new cases in 2008 [1]. Of patients with colorectal cancer, 50\% will develop liver metastasis [2]. On the other hand, primary liver cancer, which consists predominantly of hepatocellular carcinoma (HCC), is the fifth most common cancer worldwide and the third most common cause of cancer mortality [3]. Liver resection is the treatment of choice in selected patients with hepatic colorectal metastasis [4], even in recurrent cases [5]. Hepatocellular carcinoma is potentially curable by surgical resection, but surgery is the treatment of choice only for patients with localized disease [6]. The critical aspect in this procedure is to guarantee a safe margin around the tumour (R0), what is often a trade-off with 
remaining functional liver. Another key issue is reduction of bleeding and control of main veins in the liver, one of the major causes of complications. Liver resection is also critical for living-donor programmes and other non-oncologic surgical procedures.

On the other hand, technical and computational advances are constantly giving rise to new surgical concepts and techniques. By extending the surgeon's ability to plan and carry out interventions more accurately and less invasively, computer-aided surgical systems will address a crucial need to reduce medical procedure costs, and improve clinical outcome and thus the efficiency of health care delivery [7]. In this work, we focus on a system managing the organisation, image processing and display of the complex interior structures of the liver, to be used in resection procedures (hepatectomies). It allows the surgeon to orient him/herself by means of computer-generated visualizations of the anatomy, target structures and planned resection paths.

Some research work in computer-aided surgical systems for liver surgery is focussed on providing preoperative planning support [7-12]. These systems perform image segmentation and 3D reconstruction of liver, tumours and vessel structures. Based on these 3D models, they offer edition and calculation of anatomical and non-anatomical resections with user-defined security margins. User interaction is generally through two-dimensional (2D) rendered images, or even involving a 3D virtual environment [10]. Operation planning may be substantially improved by the use of such systems [8]. Nevertheless, these systems do not yet focus on presentation of information intraoperatively, and the surgeon is forced to rely on his memory and ability to translate the preoperative figures into the operative site $[8,13]$. There is still a need to effectively use the anatomical information from the $\mathrm{CT}$ in the intraoperative suite through an interactive virtual 3D model of the liver. As an example in this direction, a case study using a 3D virtual liver for navigation support has recently been reported [14].

On the other hand, recent contributions have been made to provide intraoperative guidance in liver surgery. Orientation in the liver anatomy is achieved in surgical routine using ultrasound (US). This modality is limited by a restricted field of view and decreasing practical utility as resection progresses, as it is difficult or impossible to image and interpret the structures in the resection plane once resection has started. Three-dimensional reconstruction of a US volume and an optical tracking system are the two main components of a virtual navigation system used to guide the resection in open surgery proposed by Beller et al. [15]. This system enhances the accuracy of tumour resection margins, but requires immobilisation of the liver and relative easy access to the tumour location. Another alternative is the use of an optically tracked C-Arm and laparoscope to enable an augmented view of laparoscopic images [16]. This solution provides the surgeon with advanced visual localization of hidden structures such as veins, but it produces radiation, has high cost and is bulky and only available in specialised operating rooms (OR). A third possibility is the use of laser-ranging technology to acquire the intraoperative surface of organs, and then to navigate in the registered preoperative CT volume. Preliminary results of such technology are still focussed on calibration and technical validation issues [17]. In general, all these new systems offer attractive guidance possibilities, by means of tracking and intraoperative imaging technologies. However, these technologies require delicate handling, for example for the different calibration procedures. In many cases they also introduce changes in the workflow and ergonomic aspects that could be very disruptive, for instance due to the need for a clear line of sight between the tools and the optical tracking systems. There still exists a need to efficiently enhance the safety of surgical procedures by providing the surgeon with the localization of critical structures during liver resection.

Our approach to address the clinical need for intraoperative navigation for safer liver resection is to present an interactive 3D Resection Map to the surgeon: a system for simplified and effective visualization of critical structures and the preoperatively planned resection path. This concept is somehow similar to the use of a navigation system while driving a car, or to the use of context maps in computer games $[18,19]$ but without the positioning information, i.e., without knowing the corresponding location of the tools in the map. Our strategy is to harness the rich preoperative planning information during the surgical procedure through an intuitive cartography, and without the need for any additional hardware or equipment. The system thus relies on the surgeon's capacity to perform a mental alignment between the Resection Map and the operating field. A detailed description of the design process and concept of this system is described in previous work [20].

In the consecutive series of ten patients reported herein, we prospectively validated the design and evaluated the impact of the Resection Map on operative safety and surgeon confidence for both open and laparoscopic hepatectomy. Special emphasis is given to evaluate the capacity of the surgeon to perform the mental alignment between the operating field and the Resection Map.

\section{Methods}

An explorative prospective cohort study was designed. Ten consecutive patients admitted for hepatectomy to three European hospitals from October to December 2008 were selected. The only inclusion criterion for the patients was the availability of a recent (less than 2 months) preoperative CT study with good contrast quality in veins (sufficient 
for semi-automatic segmentation of veins). Three experienced surgeons (having performed more than 25 hepatectomies each) from the hospitals were enrolled in the study. One of them had wide experience using a laparoscopic approach for this procedure, whereas the other two had only experience in open surgery.

A training and familiarization protocol was conducted in each hospital. Surgeons and assistants first received an interactive explanation of the Resection Map system. Then, an expert technician provided intraoperative support to the surgical team during the first surgery in each hospital. After that, each surgical team worked independently with the system.

The workflow for each case consisted of four steps after patient selection: CT segmentation, resection planning, intraoperative use of the Resection Map and retrieval of evaluation metrics. In this study, intraoperative updating of resection planning in the virtual $3 \mathrm{D}$ reconstructed liver was not considered. The type of resection performed for each case is described in the "Results" section.

\section{CT segmentation}

Segmentation was performed remotely with semi-automatic tools by expert engineers and verified by clinicians. CT studies were acquired with different machines and contrast protocols depending on the hospital for each patient (Table 1). Resulting image quality and resolution was thus not homogeneous among the sample population. This was an additional difficulty for a tool for segmentation and 3D reconstruction of structures, because such tools work better with known image quality and isotropic voxels (i.e., with equal dimensions in all three directions). Thus, an image decimation and interpolation process was adapted, depending on the voxel dimensions, to each dataset to obtain closer to isotropic dimensions.

A contrast-enhanced CT study was segmented into liver parenchyma, tumours, portal vein system and hepatic vein system by a two-stage semi-automatic process. First, liver parenchyma and tumours were identified with the methods described in previous work [21]. Secondly, the two vein systems were extracted with an original and pragmatic method that combines vessel enhancement filters and region-growing algorithms as described below.

Cylindrical shapes (vessels) inside the liver volume were intensified with vessel enhancement filters [22]. This was done for seven scales, i.e., seven diameters of veins (1.3-5 mm), producing seven enhanced images. The largest separable regions were identified in the image of largest scale, and a region growing algorithm was applied to the other six images of lower scale connecting the remaining smaller vessels (Fig. 1a). These regions, these vessel trees, were finally manually grouped into the two main hepatic and portal systems (Fig. 1b). If necessary, manual correction of the semi-automatic segmentation of veins was done.

CT segmentation time greatly depended on the size and quality (resolution and contrast) of the image study. The process, done with research prototype tools, took around $40 \mathrm{~min}$ (both human interaction and computer processing time) for a study of average size and good quality, and up to a few hours in cases of low contrast and resolution.

Preoperative resection planning and 3D model reconstruction

Surgical planning was performed by the surgeons using Resection Planner software designed specifically for this study. This tool enables the surgeon to load the CT study, review the segmentation results, define a resection plane and automatically generate the 3D geometries that constitute the cartography of the Resection Map (Fig. 2).

The Resection Planner software was developed on top of the open-source platform ITK-Snap [4]. In this tool, the resection plane is modelled as a smooth surface that can be deformed and adapted to complex resection scenarios. Mouse interactions are used to modify this plane in the axial, coronal and sagittal views of the CT, and interactive $3 \mathrm{D}$ visualization is provided. Surgeons have the option to "undo/redo" any change, "save" the current plane or "restore" the plane previously saved in each study.

Surgeons checked the quality of the segmentation and corrected the identification of tumours. Then they manually defined the resection plane, taking into account the visible structures in the venous phase of the CT study. The shape
Table 1 CT image acquisition parameters of the seven hepatectomy cases

\begin{tabular}{llll}
\hline Patient & CT machine & Voxel size $(\mathrm{mm})$ & Contrast \\
\hline 1 & Siemens Sensation 16 & $0.77 \times 0.77 \times 3.85$ & n.a. \\
2 & GE LightSpeed VCT & $0.70 \times 0.70 \times 1.75$ & $100 \mathrm{ml}$ 300ultra \\
3 & Toshiba Aquilon & $0.78 \times 0.78 \times 0.78$ & n.a. \\
4 & GE LightSpeed VCT & $0.69 \times 0.69 \times 1.72$ & $150 \mathrm{ml}$ VISIP 320 \\
5 & Siemens Sensation 16 & $0.69 \times 0.69 \times 3.45$ & n.a. \\
6 & GE LightSpeed VCT & $0.73 \times 0.73 \times 1.82$ & $200 \mathrm{ml}$ VISIP 320 \\
7 & Siemens Sensation 16 & $0.82 \times 0.82 \times 4.1$ & n.a. \\
\hline
\end{tabular}


Fig. 1 Manual grouping step in vessel segmentation. A Result of the automatic segmentation. (orange) vein systems finally generated by manual grouping. (Color figure online) B Hepatic (blue) and portal

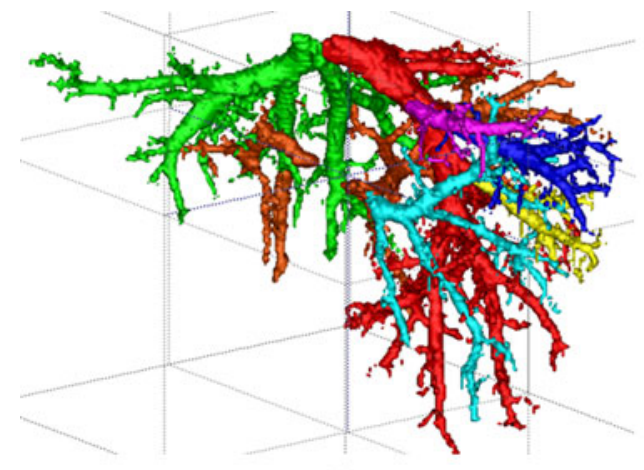

A
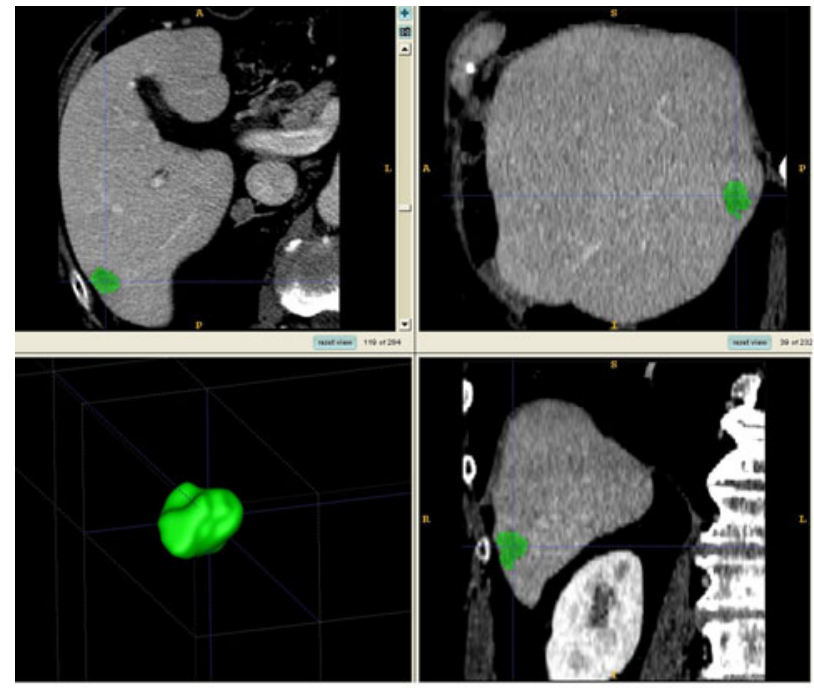

A

Fig. 2 The Resection Planner software. A Revision of the segmentation results: the tumour label (green) is made semitransparent in the three slice views to check correct location and extension. Threedimensional reconstructions are shown in the bottom left window. B

and position of the resection plane were determined with just the understanding of the vein systems and the position of the tumour, without territory analysis as proposed in other work [8]. Information from the arterial and bile duct systems was not considered in this definition. The main reason for this choice is that, compared with the venous system, arteries and bile ducts add little significance for predicting the remnant liver or navigation information during resection [20], since these intrahepatic systems mainly run in parallel to the portal system. A secondary motive is that arteries and bile ducts segmentation and 3D visualization increase the complexity of the process and the need of CT scan time and radiation to the patient.

Intraoperative assistance by the Resection Map

Surgeons performed hepatectomies following their usual standards and guidelines. The Resection Map was

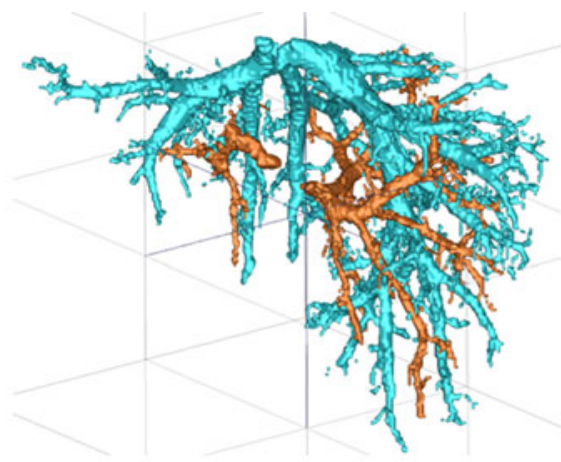

B
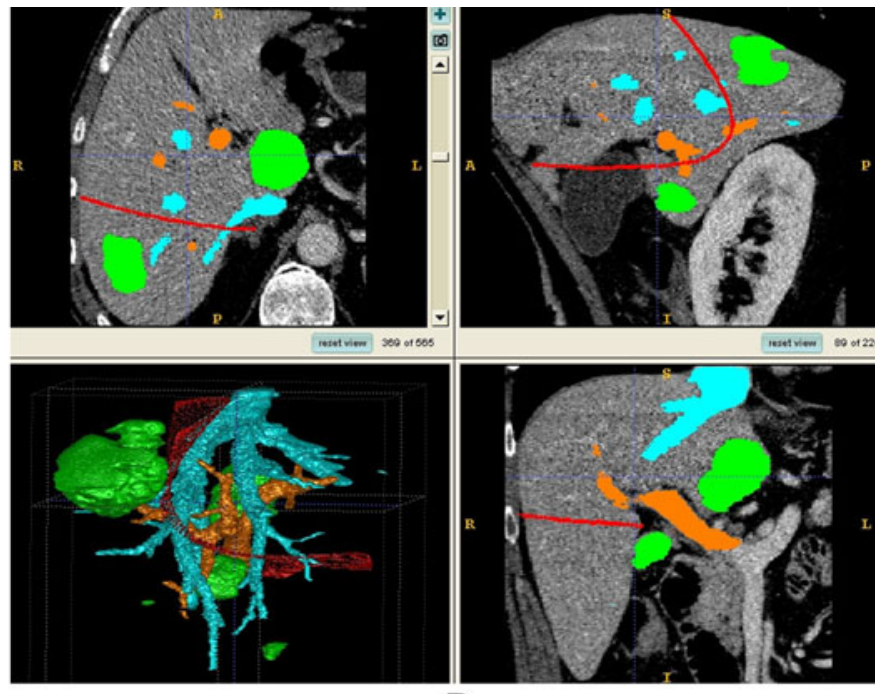

B

Definition of the resection plane (red) of a complex case with a total of six metastases (an additional segment I resection was done in this case). Hepatic veins are coded in blue, and portal veins in orange. (Color figure online)

displayed for them on a monitor as an additional source of orientation and guidance. Surgeons read the information of the Resection Map and interactively changed its visualization through voice commands to their assistants, who controlled the tool with a computer mouse interface. The interaction possibilities and contents of the Resection Map were adapted to the different phases of the surgical procedure as described below.

The Resection Map first shows an external view of the virtual liver, which is used to review the case. Surgeons can rotate this view, and activate or deactivate its elements (resection plane, tumours, liver parenchyma, or veins; Fig. 3). This view also helps the surgeon with orientation and drawing of the cauterization line over the liver's surface.

Once resection starts, the Resection Map can be changed to show an internal view of the virtual liver. This view, divided into a surgical view and a progress view, offers interactive selection and visualization of a volume in the 


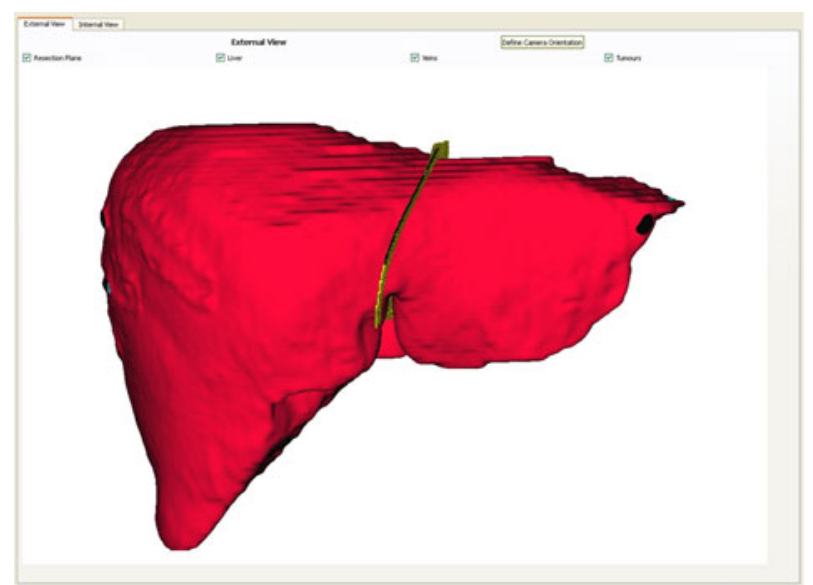

A

Fig. 3 External view of the Resection Map, showing two cases: A case 2: anatomical resection of left lobe, and $\mathbf{B}$ case 4: top view of a partial resection of segment II. Note the amount of liver preserved in this case, comparing this resection with an anatomical left lobe option. The other two tumours were locally resected (tumour located

proximity of the resection plane, depending on the resection stage. In the surgical view, structures in the neighbourhood of the resection plane are gradually shown or hidden in the direction of resection progress, as indicated by simple commands of "forward" or "backward". The 3D orientation of the virtual liver in this view is defined by the surgeon to match the orientation of the real liver and thus help the reading of the Resection Map.

The progress view, on the other hand, presents a complete overview of the entire resection plane. It displays an indicator, termed the progress window, of the volume seen in the surgical view. The progress view has a squared grid, with $1 \mathrm{~cm}$ side length, to provide a visual guide for interpretation of distances. Both the surgical and progress view can be rotated to enhance perception of 3D structures, and their elements can be activated or deactivated. For better understanding, Fig. 4 shows a practical example of the possibilities of this internal view.

To help perception of critical structures (tumours and veins cut by the resection plane) the Resection Map incorporates some visual aids. Tumours are always present in the surgical view, independent of whether they are inside the progress window; their colour becomes green in those parts that are inside the progress window. On the other hand, the vein-cutting areas, i.e., the intersection between veins and the resection plane, are highlighted in yellow. For more details about the design of the Resection Map system, the reader is referred to previous work [20].

\section{Evaluation metrics}

The objective of this study is twofold: design validation of the Resection Map system and assessment of its potential

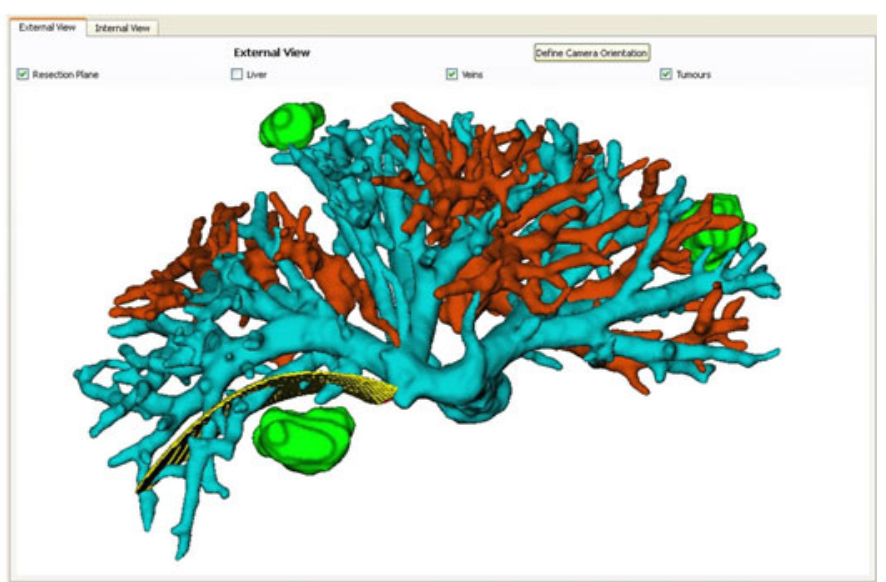

B

in segment IV) and ablated (tumour in segment VI). Parenchyma surface is removed for clarity: only vessels (blue for the hepatic and orange for the portal), resection plane (yellow) and tumours (green) are visible. (Color figure online)

impact on surgical quality and safety. The main hypothesis under study is that a surgeon can track the progress of resection using the Resection Map. By so doing, it is inferred that the surgeon will be able to better approximate the location of the tumour (reducing the risk of passing the safety margin) and to anticipate and control the next important veins (reducing the risk of bleeding). Veins, in the area where they are cut, are the landmarks displayed in yellow in the Resection Map, and are the structures used for tracking resection progress.

For each case the surgeon in charge reported in an electronic questionnaire a brief description of the surgical case, feedback on use and usability aspects related to the case (on a six-point Likert scale), and objective parameters of surgical outcome.

Design validation focussed on selected key aspects such as ease of use, efficiency in guidance (navigation aid), and quality of map and segmented information. Whereas ease of use and efficiency are standard usability quality components [23], other criteria were defined: quality of segmentation, assessed by the number of tumours that required manual correction by the surgeon; accuracy of the structures in the Resection Map, assessed by the rate of correct correspondences found between landmarks displayed in the Resection Map (see Fig. 5 for an example of landmarks) and those seen in the intraoperative US; and navigation success using the Resection Map, assessed by correct intraoperative identification of Resection Map landmarks during the resection procedure.

On the other hand, objective parameters to measure the increase of safety (amount of bleeding, and resection margin) and operating time are reported as explorative values. No comparison or statistical analysis is performed 


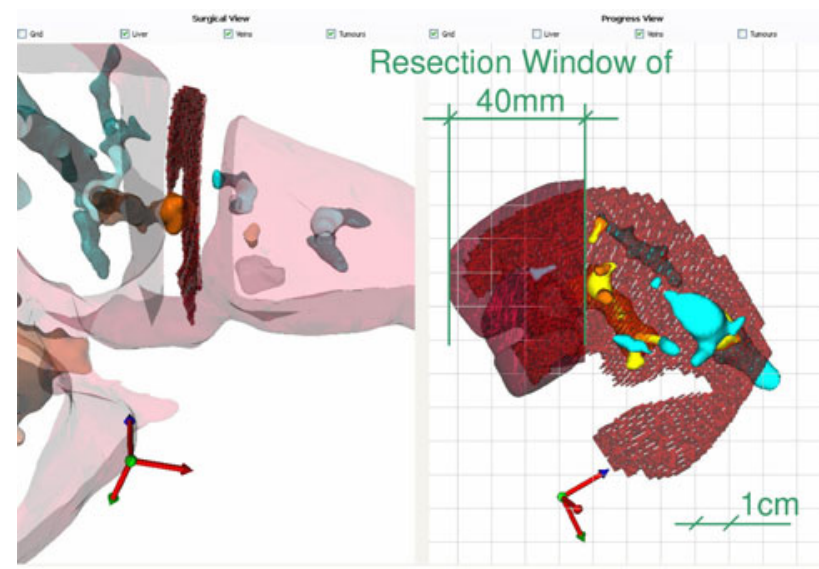

A
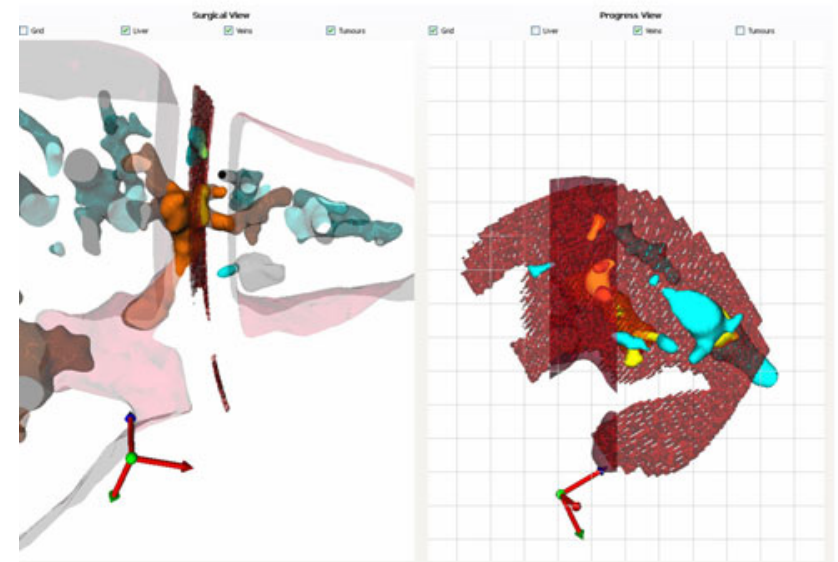

C

Fig. 4 Internal view of the Resection Map, showing some of its visualization possibilities, in case 2 (left lobe anatomical resection). Surgical and progress views are presented in the left and right part of each image, respectively. Green lines, arrows and text have been added to aid understanding. A Beginning of resection. Progress window set to $40 \mathrm{~mm}$. The surgical view therefore shows a liver slice of $40 \mathrm{~mm}$ thickness. B User reduced the progress window to $20 \mathrm{~mm}$ and advanced its position $2 \mathrm{~cm}$. Veins are deactivated in both views, and tumours are activated in the progress view; their positions are

with any control group in this exploratory cohort study, since there are many interfering factors (type of resection, surgeon, clinical condition of the patient etc.) that would prevent statistical significance being reacted. As an additional metric, surgeons were asked to rank their sense of surgical control and safety with the use of the Resection Map (on a six-point Likert scale).

\section{Results}

Integration of the Resection Map in the different operating rooms (OR) of the three hospitals was seamless. The system only required a computer connected to an intraoperative monitor. Each surgeon chose the most appropriate

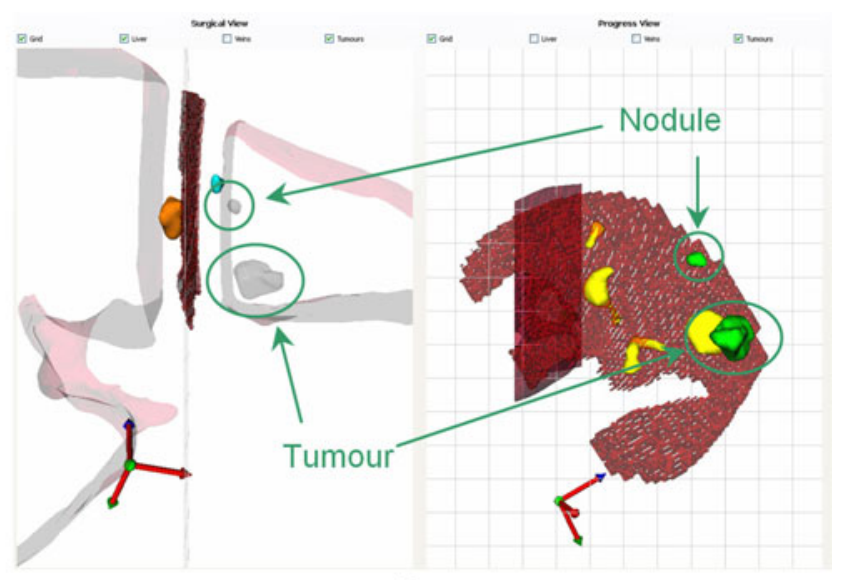

B

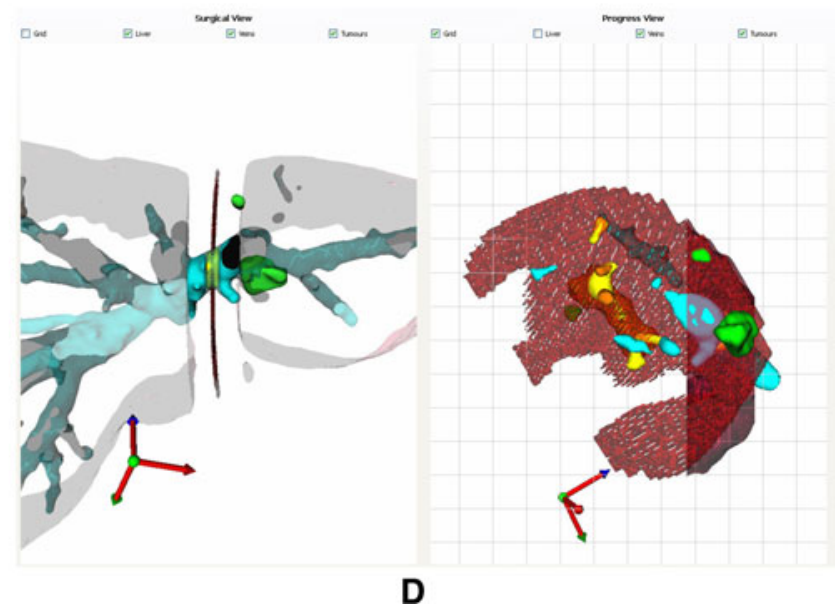

now clearly seen. Vein-cutting areas (in yellow) are now clearly seen as landmarks to track resection progress. C Progress window advanced $1 \mathrm{~cm}$. The portal three (root of segments II, III, and IV), one of the critical points in this resection, is now clearly seen in the surgical view. D Progress window advanced $4 \mathrm{~cm}$. Hepatic branch for segments II and III and relative tumour position clearly seen (tumour now in green in the surgical view since it is inside the resection window). (Color figure online)

location for this monitor in the OR, accordingly to the organization of the operation (Fig. 6).

Seven out of ten planned hepatectomies were intraoperatively assisted by the Resection Map. The three nonsuccessful cases were caused by clinical and logistical reasons: surgery cancelled due to new metastases covering the whole liver (one case) or to a new disease condition (one case), and lack of coordination in the new workflow (one case).

\section{Design validation}

Preoperatively, $7.2 \%$ ( 1 of a total of 14 ) of tumours and metastases were missed by the semi-automatic segmentation and corrected by the surgeons before the procedure. 
Fig. 5 Internal view of the Resection Map of patient 1 with manual annotations (circles and numbers) of the intersections between veins and resection plane, the yellow landmarks to identify the resection stage. The progress view (right) shows an overview of the resection plane with all five yellow landmarks and the progress window over landmarks 1 and 2. The surgical view (left) therefore focusses on veins corresponding to landmarks 1 and 2. (Color figure online)
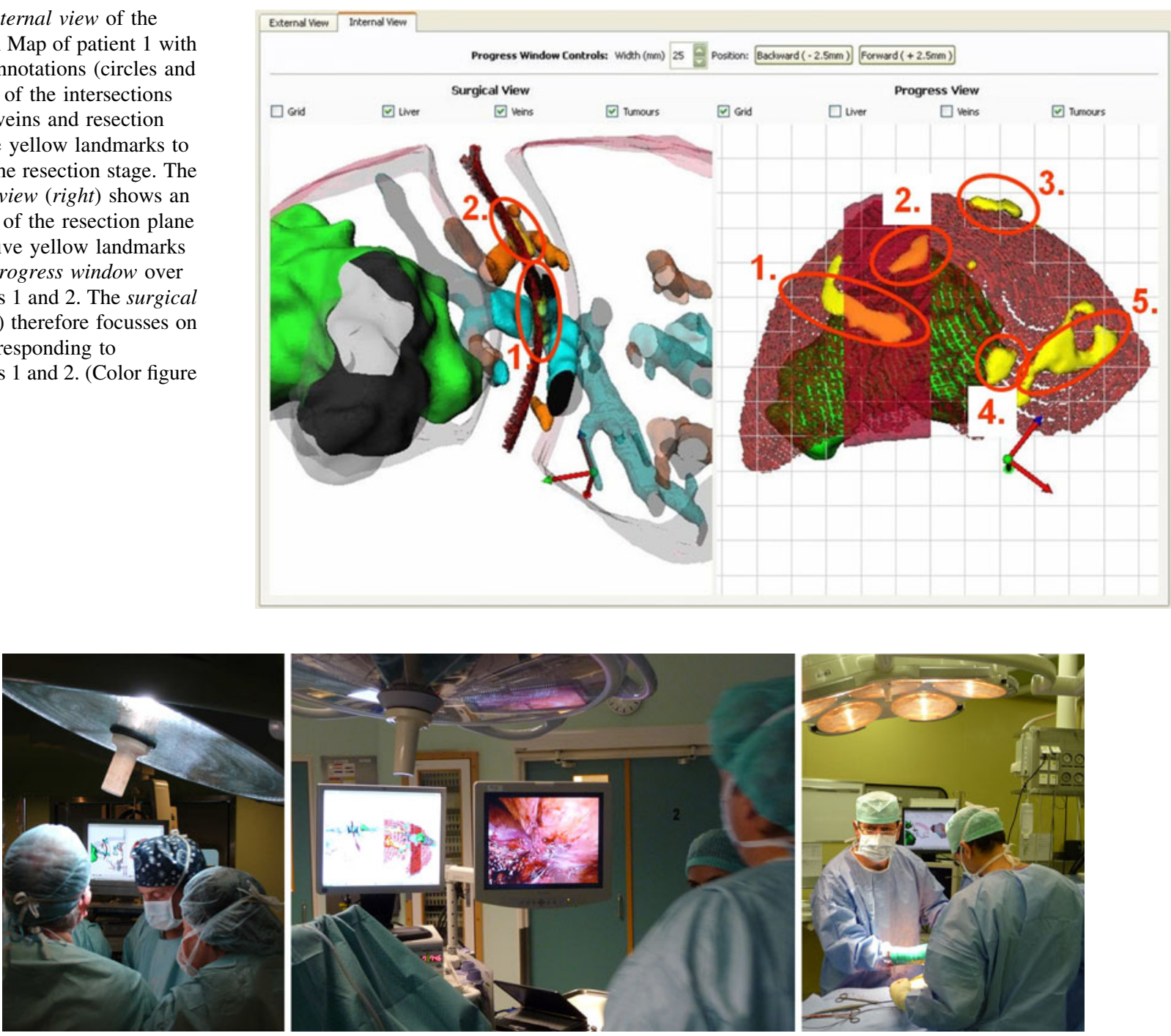

Fig. 6 The Resection Map in use in the three hospitals of the study

None of them required the boundaries of the tumour to be refined. Surgeons planned the resection in about $15 \mathrm{~min}$ and were satisfied with the result (question 1, Table 2). Intraoperatively, interaction through voice commands with an assistant was effective but had some limitations when rotating views. Surgeons found it generally easy to read the cartography of the Resection Map and find correspondences (question 2, Table 2).

The information displayed in the Resection Map intraoperatively was subjectively characterized by all surgeons as accurate. As could be expected, 3D reconstructed veins were easily identified on the intraoperative ultrasound (US) monitor. Surgeons also noted the excessive width of veins, which was a consequence of the strategy followed for CT segmentation and 3D reconstruction. The landmarks displayed in the Resection Map, the points where veins intersect the resection plane, were identified in $100 \%$ of cases when an US probe was available (Table 3).

On the other hand, surgeons were able to identify these yellow landmarks in the operating field in $70.1 \%$ of cases (Table 3). Figure 7 shows an example of one of these correspondences found in a laparoscopic procedure. The main reason for a nonidentification of a vein in the operating field was size, small veins being difficult to visualise. The use of the LigaSure Atlas (Valleylab, Boulder, CO, USA), which does not preserve vessels during liver resection, made vein identification difficult in the case of patient 7 (Table 3). Finally, intraoperative update in planned resection path for case 6 led to changes in landmarks (veins) to be found, which was also counted as non-identifications. Depth of vessel location during resection was not a determining factor: the map helped the surgeons to identify both superficial and deep vessels. 
Table 2 Design verification results (questions 1 and 2) and perceived increase of surgical quality and security (questions 3-9) in the seven surgical cases, ranked on a Likert scale from 1 (strongly disagree) to 6 (strongly agree)

\begin{tabular}{|c|c|c|c|c|c|c|c|c|}
\hline \multirow[t]{2}{*}{ Question } & \multicolumn{7}{|c|}{ Surgical case } & \multirow[t]{2}{*}{ Median } \\
\hline & 1 & 2 & 3 & 4 & 5 & 6 & 7 & \\
\hline $\begin{array}{l}\text { 1. I was satisfied with the result of the Resection Planning } \\
\text { (right shape \& position of the plane) }\end{array}$ & 5 & 6 & 6 & 6 & 5 & 5 & 6 & 6 \\
\hline $\begin{array}{l}\text { 2. It was easy to find the correspondence between the veins } \\
\text { displayed on the Map (internal view) and the veins found } \\
\text { while resecting the liver }\end{array}$ & 6 & 6 & 4 & 5 & 5 & 5 & 5 & 5 \\
\hline $\begin{array}{l}\text { 3. I understood better the case (position of the tumour, } \\
\text { resection strategy) thanks to the help of the Map } \\
\text { and the Preoperative Planning }\end{array}$ & 6 & 6 & 3 & 6 & 4 & 5 & 6 & 6 \\
\hline $\begin{array}{l}\text { 4. I increased my accuracy in drawing the cauterization line } \\
\text { over the patient's liver thanks to the help of the Map }\end{array}$ & 5 & 4 & 3 & 4 & 5 & 4 & 6 & 4 \\
\hline $\begin{array}{l}\text { 5. I could anticipate the appearance of the next vein, and to } \\
\text { estimate the location of the tumours during the resection }\end{array}$ & 6 & 5 & 5 & 5 & 4 & 4 & 5 & 5 \\
\hline $\begin{array}{l}\text { 6. I increased my control of veins, and reduced the amount } \\
\text { bleeding thanks to the help of the Map }\end{array}$ & 6 & 4 & 3 & 4 & 5 & 5 & 6 & 5 \\
\hline $\begin{array}{l}\text { 7. I increased the guarantee of a good safety margin around } \\
\text { the tumours thanks to the help of the Map }\end{array}$ & 6 & 4 & 5 & 4 & 4 & 5 & 6 & 5 \\
\hline 8. The Map increased my confidence during the resection & 6 & 6 & 4 & 6 & 5 & 5 & 6 & 6 \\
\hline 9. The Map reduced the time of the resection & 6 & 4 & 4 & 4 & 4 & 4 & 6 & 4 \\
\hline
\end{tabular}

Table 3 Rate of correct identification by the surgeon of landmarks (vein-cutting areas) displayed in the Resection Map

\begin{tabular}{llll}
\hline Patient & $\begin{array}{l}\text { Surgeon } \\
\text { identification in US }\end{array}$ & $\begin{array}{l}\text { Landmark } \\
\text { the operating field }\end{array}$ \\
\hline 1 & 1 & n.a. & $4 / 5$ \\
2 & 2 & $4 / 4$ & $3 / 4$ \\
3 & 3 & n.a. & $5 / 7$ \\
$4^{\mathrm{a}}$ & 2 & $5 / 5$ & $4 / 5$ \\
& & $2 / 2$ & $2 / 2$ \\
5 & 2 & $1 / 1$ & $1 / 1$ \\
6 & 2 & $3 / 3$ & $2 / 3$ \\
7 & 1 & n.a. & $1 / 4$ \\
Total & & $100 \%(15 / 15)$ & $70.1 \%(22 / 31)$ \\
\hline
\end{tabular}

Landmarks were identified both in the US and in the operating field n.a. US probe not available during surgery

${ }^{a}$ Patient 4 had two local resections to remove two different tumours

\section{Surgical outcome}

Intraoperative bleeding, surgical time, resection margin and a brief description of the resection done in each case are presented in Table 4. Subjective perception of the increase of safety and security is reported in Table 2. Surgeons strongly agreed that the Resection Map increased their confidence, and that they understood better the resection case. In comparison, reduction of operative time and increase of accuracy while drawing the cauterization line were the aspects with the smallest subjective score (4, "slightly agree"). Surgeons agreed that they could anticipate the location of and increase the control of veins. Finally, they also agreed that use of the system increased assurance of a good safety margin around tumours.

\section{Discussion}

Surgical demand exists for computer-aided surgical systems for both open and laparoscopic liver resections. Surgeons expect orientation and visualization support during operations that allow for more accurate and secure execution of the planned operation, especially in non-anatomical resections. The pragmatic solution of a Resection Map fulfils part of this need without locating any additional equipment in the OR, as proposed by other recent approaches [15-17].

The scope of the present study was focussed on the capacity of the surgeon to track resection progress by using a Resection Map. In our experimental design, and with limited resources (only 3 months of time), we tried to maximize the number of experienced surgeons from different hospitals using the tool. Despite some lack of coordination in one case, the integration of the new workflow involving the Resection Map was seamless. The number of surgical cases we finally managed to process was small, but each of them provided several test cases of vein identification, and all were deemed successful 
Fig. 7 Intraoperative use of the Resection Map in patient 2 while finding correspondences between the Resection Map and the US, or between the Resection Map and the surgical field. A Surgeon mapping the structures seen in the external view to the US image. B Resection Map to laparoscopic view correspondence of the portal root at the point where it divides into the branches of segments II, III and IV

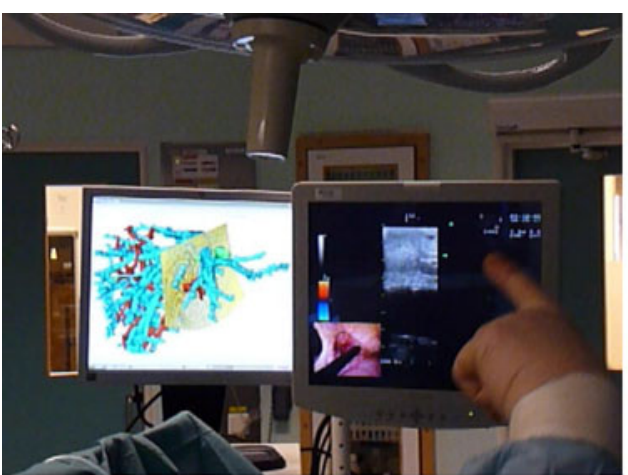

A

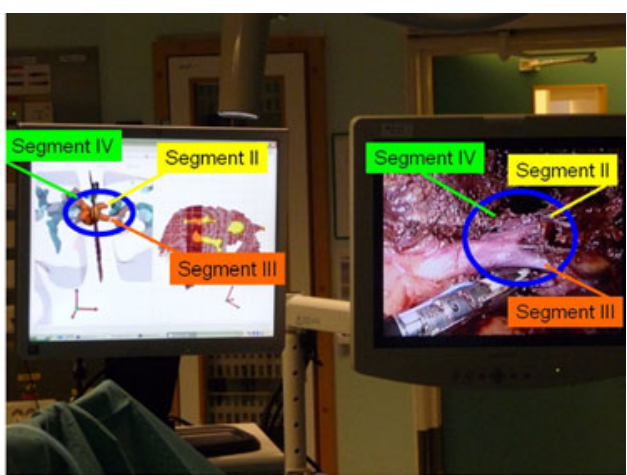

B

Table 4 Type of resection and surgical outcome for each case

\begin{tabular}{llllllll}
\hline Patient & Tumours & Technique & Location & Resection & Bleeding (ml) & $\begin{array}{l}\text { Tumour } \\
\text { margin }(\mathrm{cm})\end{array}$ & $\begin{array}{l}\text { Surgery } \\
\text { time }(\mathrm{min})\end{array}$ \\
\hline 1 & 1 & Open & S.VI & S.VI & 400 & $1.0(\mathrm{R} 0)$ \\
2 & 1 & Laparoscopic & S.II & Left lobe & Minimal & $1.5(\mathrm{R} 0)$ \\
3 & 6 & Open & Right hemiliver, S.I & Right hemiliver + S.I & 450 & $1.6(\mathrm{R} 0)$ & 192 \\
4 & 3 & Lap. + RFA & S.II, IV, VI & Partial S.II + Partial S.IV & 150 & $0.6 \& 0.8(\mathrm{R} 0)$ & 131 \\
5 & 1 & Laparoscopic & S.VII & Partial S.VII & 700 & $0.1(\mathrm{R} 0)$ & 236 \\
6 & 1 & Laparoscopic & S.VII & Partial S.VII & Minimal & $0.2(\mathrm{R} 0)$ & 101 \\
7 & 1 & Laparoscopic & S.V-VI & S.V-VI & 90 & $0.8(\mathrm{R} 0)$ & 130 \\
\hline
\end{tabular}

$R F A$ radiofrequency ablation

demonstrations of improved surgical navigation. It has to be highlighted that, in case 7, the tool helped the surgeon with the adoption of the laparoscopic approach in liver resection (surgeon 1), and that even a very experienced laparoscopic surgeon (surgeon 2) reported an increase of his sense of control and confidence.

Two main characteristics are required for a map to be useful and effective: the accuracy of the information displayed and the ease of reading this information by the user. The map then provides guidance and orientation through consecutive identification of structures and landmarks. This is the added value of the Resection Map to assist in hepatectomies. Surgeons were easily able to make a mental mapping of the structures displayed by the Resection Map to the intraoperative US images $(100 \%$ success rate of landmark identification when the US probe was available; Table 3). The segmentation and 3D reconstruction processes were therefore deemed accurate.

Interaction with the Resection Map through an assistant was effective. Nevertheless, direct control by the surgeon, with a sterilised touch-screen for example, might be more efficient. Surgeons were also able to identify and recognise the main veins found during the resection $(70.1 \%$ successful rate; Table 3). The Resection Map enhanced their capacity to orient themselves, and they experienced an increase in confidence and sense of control over the main veins and safety margin of tumours (Table 2). Nevertheless, an effective increase of safety has not been demonstrated in this initial exploratory trial. This requires a prospective and randomized trial, which falls outside the scope of the present study.

The Resection Map can be understood as an intuitive intraoperative solution to visualize the CT information of the patient during surgery. It is therefore limited by the available quality of this information. In our study we successfully generated a Resection Map from low-resolution studies (Table 1) thanks to the expertise of the engineers involved. Nevertheless, current state of CT technology, with multislice machines with 64 slices or more, and existing robust CT segmentation algorithms [9] will gradually be adopted by hospitals and make automatic virtual model generation an easy, common practice in clinical routine. Some efforts are also needed to integrate picture archiving and communication systems (PACS) and what we could call the surgical information system to enable smooth introduction of these intraoperative solutions into hospitals. 
The Resection Map bridges the gap existing in current liver planning systems [8-12] of effective translation of preoperative analysis into intraoperative guidance. Planning systems can also be used in the OR [24], but they are not designed to fulfil intraoperative requirements. The Resection Map, on the other hand, is a virtual navigation environment without the relative positioning of surgical tools, which can be provided in an immobilised liver at the cost of additional hardware and a more complex surgical workflow [15-17]. There is no solution yet that copes with intraoperative deformations or that is able to track and adapt. A solution such as the Resection Map relies not on additional hardware but on the surgeon's capacity to read and map the cartography displayed to them. We therefore think that this is a pragmatic solution that solves part of the intraoperative navigation need quite efficiently.

We believe that the Resection Map could be very helpful for the education of inexperienced liver surgeons, for the adoption of a laparoscopic approach and for complex cases of an experienced surgeon. The tool could even substitute some of the uses of intraoperative US, such as the identification of key vessels that are going to be cut during resection. Nevertheless, US will still be required for verification of the position and size (and possible growth) of known tumours, and the identification of new ones.

Future work will be directed towards full automation of the Resection Map system, and towards comprehensive study to measure the increase of safety during hepatectomies and verify the hypothetical benefits of the tool stated above. Technically, the tool should include the capability to update the resection plane intraoperatively after finding new metastases. Also, in order to enhance interactivity and navigation experience, future research should address the challenge of incorporating tool positioning information and adapting to the deformations of the liver while minimizing disturbance to the surgical workflow [25].

\section{Conclusion}

The Resection Map is a simple and pragmatic solution conceived to enhance the safety of liver resection. Its integration into the operating room was seamless, and preliminary results showed a perceived increase in safety and confidence of the surgeon. Other possible applications of this technology are easier adoption of a laparoscopic approach by surgical teams, easier implantation of a livingdonor programme and education of inexperienced surgeons.

Acknowledgements This work is part of the ARIS*ER Marie Curie Research Training Network (MRTN-CT-2004-512400), which is funded by the $6^{\text {th }}$ Framework Programme of the European Commission. We especially acknowledge E. Samset for support and excellent management and coordination, A. Jalote-Parmar for her contributions at the early stage of this research, A. Serrablo for his enthusiasm and engagement, and our partners and hospital staff for help and support in the development and validation work.

Disclosures Authors Pablo Lamata, Félix Lamata, Valentin Sojar, Piotr Makowski, MSc Laurent Massoptier, Sergio Casciaro, MSc Wajid Ali, Thomas Stüdeli, Jérôme Declerck, Ole Jackov Elle, and Björn Edwin have no conflicts of interest or financial ties to disclose.

Open Access This article is distributed under the terms of the Creative Commons Attribution Noncommercial License which permits any noncommercial use, distribution, and reproduction in any medium, provided the original author(s) and source are credited.

\section{References}

1. Jemal A, Siegel R, Ward E, Hao Y, Xu J, Murray T, Thun MJ (2008) Cancer statistics, 2008. CA Cancer J Clin 58:71-96

2. Honore C, Detry O, Deroover A, Piront P, Polus M, Honore P, Meurisse M (2008) When should we resect colorectal liver metastases? Rev Med Liege 63:595-599

3. El-Serag HB, Rudolph KL (2007) Hepatocellular carcinoma: epidemiology and molecular carcinogenesis. Gastroenterology 132:2557-2576

4. Grundmann RT, Hermanek P, Merkel S, Germer CT, Grundmann RT, Hauss J, Henne-Bruns D, Herfarth K, Hermanek P, Hopt UT, Junginger T, Klar E, Klempnauer J, Knapp WH, Kraus M, Lang H, Link KH, Lohe F, Merkel S, Oldhafer KJ, Raab HR, Rau HG, Reinacher-Schick A, Ricke J, Roder J, Schafer AO, Schlitt HJ, Schon MR, Stippel D, Tannapfel A, Tatsch K, Vogl TJ (2008) Diagnosis and treatment of colorectal liver metastases-workflow. Zentralbl Chir 133:267-284

5. Shaw IM, Rees M, Welsh FK, Bygrave S, John TG (2006) Repeat hepatic resection for recurrent colorectal liver metastases is associated with favourable long-term survival. Br J Surg 93:457-464

6. Bryant R, Laurent A, Tayar C, van Nhieu JT, Luciani A, Cherqui D (2008) Liver resection for hepatocellular carcinoma. Surg Oncol Clin North Am 17:607-633, ix

7. Marescaux J, Clement JM, Tassetti V, Koehl C, Cotin S, Russier Y, Mutter D, Delingette H, Ayache N (1998) Virtual reality applied to hepatic surgery simulation: the next revolution. Ann Surg 228:627-634

8. Lang H, Radtke A, Hindennach M, Schroeder T, Fruhauf NR, Malago M, Bourquain H, Peitgen HO, Oldhafer KJ, Broelsch CE (2005) Impact of virtual tumor resection and computer-assisted risk analysis on operation planning and intraoperative strategy in major hepatic resection. Arch Surg 140:629-638

9. Soler L, Delingette H, Malandain G, Montagnat J, Ayache N, Koehl C, Dourthe O, Malassagne B, Smith M, Mutter D, Marescaux J (2001) Fully automatic anatomical, pathological, and functional segmentation from CT scans for hepatic surgery. Comput Aided Surg 6:131-142

10. Reitinger B, Bornik A, Beichel R, Schmalstieg D (2006) Liver surgery planning using virtual reality. IEEE Comput Graph Appl 26:36-47

11. Meinzer H-P, Thorn M, Cardenas CE (2002) Computerized planning of liver surgery-an overview. Comput Graph 26:569_ 576

12. Sojar V, Stanisavljevic D, Hribernik M, Glušic M, Kreuh D, Velkavrh U, Fius T (2004) Liver surgery training and planning in 3D virtual space. Proc CARS 2004 1268:390-394

13. Jalote-Parmar A, Pattynama $P$, de Ridder H, Goossens R, Freudenthal A, Samset E (2007) Surgical workflow analysis: 
identifying user requirements for surgical information systems. In: Pikaar R, Ernst K, Settels (eds) Diversity in ergonomics. Elsevier, Oxford, pp 229-241

14. Mutter D, Dallemagne B, Bailey C, Soler L, Marescaux J (2009) $3 \mathrm{D}$ virtual reality and selective vascular control for laparoscopic left hepatic lobectomy. Surg Endosc 23:432-435

15. Beller S, nerbein M, Eulenstein S, Lange T, Schlag PM (2007) Feasibility of navigated resection of liver tumors using multiplanar visualization of intraoperative 3-dimensional ultrasound data. Ann Surg 246:288-294

16. Feuerstein M, Mussack T, Heining SM, Navab N (2008) Intraoperative laparoscope augmentation for port placement and resection planning in minimally invasive liver resection. IEEE Trans Med Imag 27:355-369

17. Cash DM, Miga MI, Glasgow SC, Dawant BM, Clements LW, Cao Z, Galloway RL, Chapman WC (2007) Concepts and preliminary data toward the realization of image-guided liver surgery. J Gastrointest Surg 11:844-859

18. Jalote-Parmar A, Pattynama PMT, Van der Plas AP, Heer P (2007) Towards intuitive surgical interfaces: strategies from video games. In: Casciaro S, Distante A (eds) New technology frontiers in minimally invasive therapies. Lupiensis Biomedical pp 101-111

19. Stüdeli $T$ (2008) Surgical navigation during minimally invasive procedures. In: Casciaro E, Samset E (eds) Minimally invasive technologies and nanosystems for diagnosis and therapies. Lupiensis Biomedical, Lecce, Italy, pp 177-186

20. Lamata P, Jalote-Parmar A, Lamata F, Declerck J (2008) The Resection Map, a proposal for intraoperative hepatectomy guidance. Int J CARS 3:299-306

21. Massoptier L, Casciaro S (2008) A new fully automatic and robust algorithm for fast segmentation of liver tissue and tumors from CT scans. Eur Radiol 18:1658-1665

22. Frangi AF, Niessen WJ, Hoogeveen RM, van WT, Viergever MA (1999) Model-based quantitation of 3-D magnetic resonance angiographic images. IEEE Trans Med Imag 18:946-956

23. Nielsen J (1995) Usability engineering. Morgan Kaufmann. San Francisco, CA, USA

24. Grenacher L, Thorn M, Knaebel HP, Vetter M, Hassenpflug P, Kraus T, Meinzer HP, Buchler MW, Kauffmann GW, Richter GM (2005) The role of 3-D imaging and computer-based postprocessing for surgery of the liver and pancreas. Rofo 177:12191226

25. Lamata P, Morvan T, Reimers M, Samset E, Declerck J (2009) Addressing shading-based laparoscopic registration. In: Dössel O, Schlegel WC (eds) WC 2009, IFMBE proceedings 25/VI, pp 189-192 (for further reference: http://www.springerlink.com/ content/j76732394677363w/?p=4693b05106ef4b109c37bf9e11 ae0fd8\&pi=0) 